Raucherentwöhnung

\section{Finanzieller Anreiz erhöht Erfolgsquote}

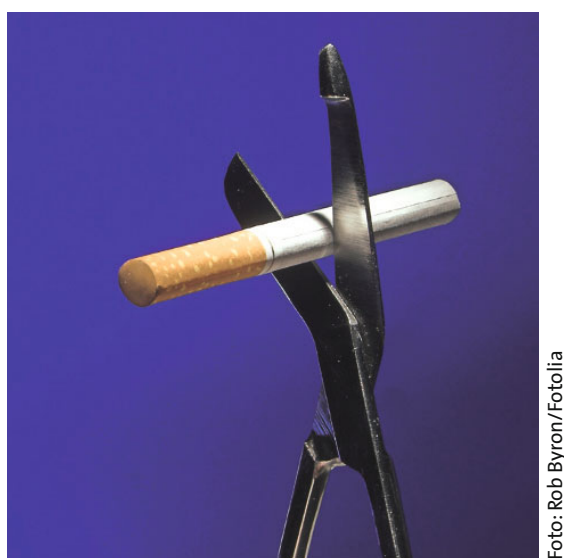

Rauchstopp: Mit Geld dazu motivieren?

— Programme zur Raucherentwöhnung haben langfristig meist nur einen bescheidenen Erfolg. Durchaus erwägenswert wäre es nach dem Ergebnis einer neuen Studie, für eine erfolgreiche Entwöhnung eine finanzielle Belohnung in Aussicht zu stellen. In der Studie wurde dadurch die Zahl der längerfristig Abstinenten fast verdreifacht.

\section{Keine Frage des Einkommens}

Knapp 900 Angestellte unterschiedlicher Nationalität - die finanziell nicht schlecht gestellt waren, wie Professor Dr. Claus Vogelmeier aus Marburg betonte-, wurden in zwei Gruppen randomisiert: Die eine Gruppe erhielt nur Informationen zum Raucherentwöhnungsprogramm. Der anderen Gruppe wurden zusätzlich 100 US-Dollar versprochen für die erfolgreiche Beendigung des Programms, weitere 250 Dollar für die Beendigung des Rauchens innerhalb von sechs Monaten nach Einschluss (bestätigt durch einen biochemischen Test) und nochmals 400 Dollar für die Abstinenz über weitere sechs Monate.

Die Ergebnisse sprechen für sich: Neun oder zwölf Monate nach Einschluss in die Studie waren nur 5\% der Kontrollpersonen abstinent, aber fast $15 \%$ der Personen, die belohnt wurden. Kontinuierlich abstinent für 15 oder $18 \mathrm{Mo-}$ nate waren $3,6 \%$ vs. $9,4 \%$.

Zweifelhaftes Diagnosekriterium

\title{
Wie relevant ist eine Tracheobronchomalazie?
}

- Eine sogenannte Tracheobronchomalazie, das heißt ein Kollaps der Luftwege beim Ausatmen, kann bei Patienten mit chronischen Atemwegserkrankungen häufig nachgewiesen werden. Allerdings wird die gängige Definition der Tracheobronchomalazie, eine Reduktion der Querschnittfläche der Atemwege um mehr als 50\% beim Ausatmen, durch aktuelle Untersuchungen infrage gestellt: Mehr als drei Viertel der gesunden Probanden erfüllten die diagnostischen Kriterien einer Tracheobronchomalazie.

\section{Messwerte mit Vorsicht interpretieren}

An der Untersuchung beteiligten sich 51 Personen im Alter von 25 bis 75 Jahren mit normaler Lungenfunktion, die Nichtraucher waren und keine Risikofaktoren einer Tracheobronchomalazie hatten. Die Trachea wurde bei maximaler Inspiration und während forcierter Exspiration per CT vermessen. Im Schnitt reduzierte sich bei den Probanden der Querschnitt der oberen Trachea beim Ausatmen um 54\% und der der unteren Trachea um 56\%, berichtete
Professor Dr. Gerhard Mostbeck aus Wien. Solche Messergebnisse sollten also mit Vorsicht interpretiert werden. Allerdings wurde bei keinem einzigen Probanden eine sichelförmige Konfiguration der Trachea nachgewiesen, was als Zeichen einer manifesten Tracheobronchomalazie gewertet wird.

Zum Vergleich: In einer ebenfalls aktuellen Studie bei 71 Patienten mit COPD, von denen gut die Hälfte eine Malazie der Luftwege hatten, war ein halbmondförmiger Kollaps der Luftwege die häufigste morphologische Konfiguration. Nach dem Ergebnis der Studie war die Häufigkeit einer Tracheobronchomalazie bei COPD-Patienten aller Schweregrade ähnlich. Auch ein Lungenemphysem oder Bronchiektasen beeinflussten die Tracheobronchomalazie-Rate nicht, es gab aber Korrelationen mit der Bronchialwanddicke.

Eine hohe Prävalenz einer Tracheobronchomalazie wurde in einer weiteren aktuellen Studie bei Patienten mit cystischer Fibrose (CF) nachgewiesen. Bei dynamischer $\mathrm{CT}$ während eines Hustenstoßes zeigten 69\% der Patienten eine Tracheobronchomalazie.

\section{Impulsoszillometrie}

\section{Eine gute Ergänzung zum Spirometer?}

- Die Impulsoszillometrie - als Ergänzung zur Spirometrie bei fehlender Ganzkörperplethysmografie - gewinnt bei der Diagnostik von Atemwegserkrankungen wieder an Bedeutung, so die Einschätzung von Professor Dr. Helgo Magnussen aus Großhansdorf.

Der Vorteil der Oszillationsmethoden ist die Anwendung bei Ruheatmung und die schnelle und einfache Durchführbarkeit. Vor allem bei Kindern und bei Patienten, die schlecht kooperieren, ist die oszillatorische Messung des Atemwegswiderstands günstig, da auf kom- plizierte Atemmanöver verzichtet werden kann. Auch zur Frühdiagnostik und zum Screening könnte das Verfahren laut Magnussen seinen Platz finden. Nachteil der Technik ist die komplexe Interpretation der Messergebnisse. Das Verfahren ist bisher kein Standard.

Autor: Roland Fath

Quelle: PneumoUpdate, Wiesbaden, 20./21. November 2009 